\title{
DOI: https://doi.org/10.24297/jam.v20i.8953
}

\section{On Regional Boundary Gradient Strategic Sensors In Diffusion Systems}

\author{
Raheam A. Al-Saphory ${ }^{1 . *}$ and Ahlam Y. Al-Shaya ${ }^{2}$ \\ 1,2 Department of Mathematics, College of Education For Pure Sciences, Tikrit University, Iraq. \\ saphory@tu.edu.iq, Ahlam.Y.Abdullah00200@st.tu.edu.iq
}

\begin{abstract}
:
This paper is aimed at investigating and introducing the main results regarding the concept of Regional Boundary Gradient Strategic Sensors (RBGS-sensors) the in Diffusion Distributed Parameter Systems (DDPSystems). Hence, such a method is characterized by Parabolic Differential Equations (PDEs) in which the behavior of the dynamic is created by a Semigroup $\left(S_{\Delta}(t)\right)_{t \geq 0}$ of Strongly Continuous type (SCSG) in a Hilbert Space (HS) . Additionally, the grantee conditions which ensure the description for such sensors are given respectively to together with the Regional Boundary Gradient Observability (RBG-Observability) can be studied and achieved . Finally, the results gotten are applied to different situations with altered sensors positions are undertaken and examined.
\end{abstract}

Keywords: WRBG-Obsevability, ERBG-Obsevability, RBGS-Sensors, DDP-Systems.

\section{Introduction}

The Observation Problem [1-3] is one of the most important notion in the analysis of DDP-Systems was attracted the attention of many researchers [4-7]. In various cases, one may interest in the cognition of the state of a PDEs system on a sub region $\mathfrak{F}$ of internal and boundary the domain $\mho$ in a unbounded interval [8-18] or bounded time [19-23].

The investigation of this notion is incited by specific Physical Problem, in Thermic, Mechanic, Environment, for example some physical problems concern the determination of laminar flux conditions, developed in steady state by vertical uniformly heated plate [24-27].

This approach can be applied to find the unknown boundary convective condition on the front face of the active plate, as in [26]. The reconstruction is based on knowledge of the dynamical system via measurement information given by internal sensors type pointwise $\left(\mathfrak{b}_{1}, \mathfrak{b}_{2}\right)$ (that means by the thermocouples for instance see (Figure 1).

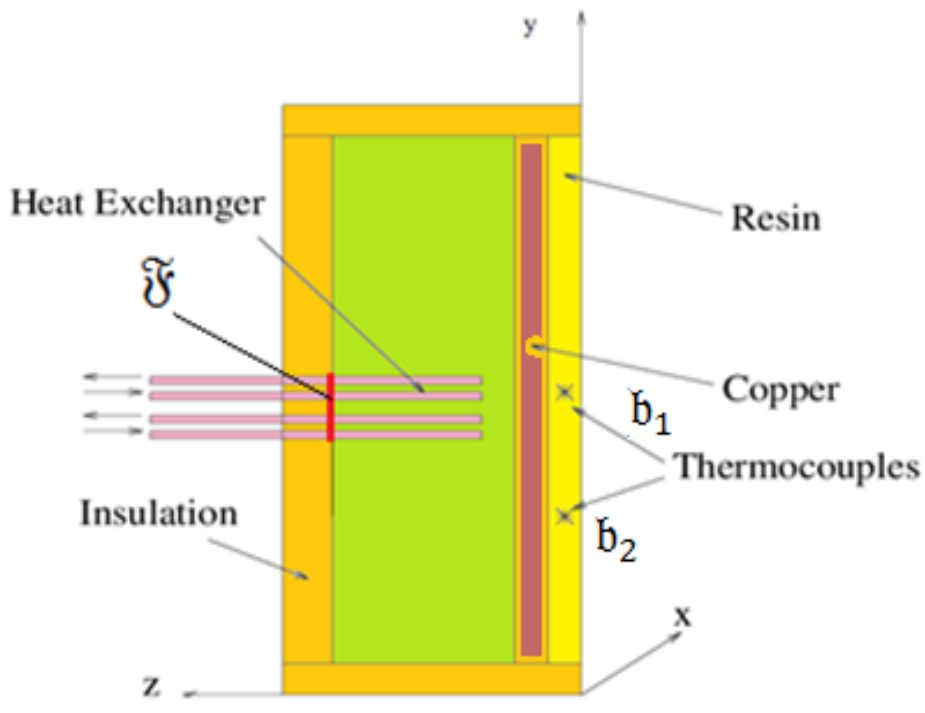

Fig.1:Real heated plate diffusion.

Thence, this study designed at giving the required conditions of the RBGS-Sensors in this region, that builds RBG-State. Thus, the main reasons for presenting this notion are: Firstly it makes cognition for the usual observer concept closer to actual world quandaries, Secondly it can be introduced and explore the main results concerned to the DDP-Systems [24-26 ] in connection with RBGS - Sensors.This job is arranged in the following: 
Certain definitions with identification of the ERBG - Obsevability for exactly case and WRBG-Obsevability for weakly case, are given in the next section. Section three introduces most for the required ailments to RBGSSensors and a reformation process is developed to come across the internal state region to the boundary. Later, several applications for sensors positions in regions of rectangular types are presented and illustrated.

\section{RBG-Observability in DDP-Systems}

The current section invests to study the notion of RBG-Observability in DDP-Systems. It makes certain important outcomes concerning this notion.

\subsection{Preliminaries Considerations Of The System}

The following assumptions are to be given

- $\mho$ stay Open and Bounded in $\mathcal{R}^{n}$, is the space domain with smooth boundary $\partial \mho$.

- $\mathfrak{F}$ remains a sub-boundary on $\partial \mho$.

- $[O, \mathcal{T}], \mathcal{T}>O$ stand to a space-time interval cylinder.

- The $H S s$ with $\mathbb{W}, \mathbb{U}$ and $\mathbb{Y}$ are separable where $\mathbb{W}$ is the space of the state $w, \mathbb{U}=\mathcal{L}^{2}\left(0, \mathcal{T}, \mathcal{R}^{p}\right)$ is the space of the input $u$ and $\mathbb{Y}=\mathcal{L}^{2}\left(0, \mathcal{T}, \mathcal{R}^{q}\right)$ is the space of output $\mathcal{Y}[16]$.

- Reflected DDP - System described by the following PDES

$$
\left\{\begin{array}{lr}
\frac{\partial w}{\partial t}(\zeta, t)=\Delta w(\zeta, t)+B u(t) & \Pi_{\mathcal{T}} \\
w(\zeta, 0)=w_{0}(\zeta) & \bar{\mho} \\
\frac{\partial w}{\partial v}(\mu, t)=0 & \bar{\Xi}_{\mathcal{T}}
\end{array}\right.
$$

where $\left.\Pi_{\mathcal{T}}=\mho \times\right] 0, \mathcal{T}\left[, \Xi_{\mathcal{T}}=\partial \mho \times\right] 0, \mathcal{T}[, \zeta \in \mho, \mu \in \partial \mho, t \in[0, \mathcal{T}]$, and $(\zeta, t) \in \mho \times] 0, \mathcal{T}[,(\mu, t) \in \partial \mho \times$ ] $0, \mathcal{T}\left[,(\zeta, 0) \in \bar{\mho}\right.$, wherever $\bar{\mho}$ represents $\mho$ closure and $\frac{\partial w}{\partial v}$ indicates the derivative of normal vector $v$ on $\partial \mho$. Then $D D P$ - System remains augmented with the measurement function

$$
y(., t)=\mathcal{C} w(., t)
$$

where,

- $\triangle$ stays an operator, linear and differential of second order type, in which is produced a SCS - group $\left(S_{\Delta}(t)\right)_{t \geq 0}$ on $H S$ may be symbolized by $\mathbb{W}=H^{1}(\bar{\mho})$ such that it is self adjoint through resolvent of compact type.

- So the operators $B \in \mathcal{L}\left(\mathcal{R}^{p}, \mathbb{W}\right)$ and $\mathcal{C} \in \mathcal{L}\left(\mathbb{W}, \mathcal{R}^{q}\right)$ be dependent on the of sensors (actuators) construction [6]. Thus the reflected DDP-Systems (1) possesses a solution of unique kind [1-3] illustrated in the subsequent form

$$
w(\zeta, t)=S_{\Delta}(t) w_{0}(\zeta)+\int_{0}^{t} S_{\Delta}(t-\tau) B u(\tau) d \tau
$$

- The problematic underlies, in what way to realize satisfactory conditions of RBGS - Sensors on specified subboundary $\mathfrak{F}$.

- Thence, the operator is defined

$$
\mathcal{K}: w \in \mathbb{W} \rightarrow \mathcal{K} w=\mathcal{C} S_{\Delta}(.) w \in \mathbb{Y}
$$

And, the adjoint operator of $\mathcal{K}$ indicates by $\mathcal{K}^{*}$ identified by

$$
\mathcal{K}^{*} \mathcal{Y}^{*}=\int_{0}^{t} S_{\Delta}^{*}(s) C^{*} y^{*}(s) d s
$$

- Consider the gradient operator

$$
\left\{\begin{array}{l}
\nabla: H^{1}(\mho) \rightarrow\left(H^{1}(\mho)\right)^{n} \\
w \rightarrow \nabla w=\left(\frac{\partial w}{\partial \zeta_{1}}, \ldots, \frac{\partial w}{\partial \zeta_{n}}\right)
\end{array}\right.
$$


and the adjoint of $\nabla$ indicated by $\nabla^{*}$ is given as

$$
\left\{\begin{array}{l}
\nabla^{*}:\left(H^{1}(\mho)\right)^{n} \rightarrow H^{1}(\mho) \\
w \rightarrow \nabla_{w}^{*}=v
\end{array}\right.
$$

whereas $v$ is a solution of the Dirichlet problem

$$
\left\{\begin{array}{l}
\Delta v=-\operatorname{div}(w) \text { in } \mho \\
\partial v / \partial v=0 \text { in } \partial \mho
\end{array}\right.
$$

- Then Operator of Trace type of zero-order is offered by

$$
\gamma_{0}: H^{1}(\mho) \rightarrow H^{1 / 2}(\partial \mho)
$$

Therefore, the propagation of the trace operator where is described via

$$
\gamma:\left(H^{1}(\mho)\right)^{n} \rightarrow\left(H^{1 / 2}(\partial \mho)\right)^{n}
$$

with the related Adjoint Operators $\gamma_{0}^{*}$ and $\gamma^{*}$.

- On behalf of a sub-boundary $\mathfrak{F} \subset \partial \mho$, we take into account a gradient restriction operator

$$
\chi_{\mathfrak{F}}:\left(H^{1 / 2}(\partial \mho)\right)^{n} \rightarrow\left(H^{1 / 2}(\mathfrak{F})\right)^{n}
$$

and

$$
\widetilde{x}_{\mathfrak{F}}: H^{1 / 2}(\partial \mho) \rightarrow H^{1 / 2}(\mathfrak{F})
$$

where the adjoints are correspondingly presented by $\mathcal{X}_{\mathfrak{F}^{\prime}}^{*} \widetilde{x}_{\mathfrak{F}}^{*}$.

- If $\omega$ remains a subregion of $\mho$, then $X_{\omega}$ is an operator specified by

$$
x_{\omega}:\left\{\begin{array}{c}
\left(H^{1}(\mho)\right)^{n} \rightarrow\left(H^{1}(\omega)\right)^{n} \\
w \rightarrow x_{\omega} w=\left.w\right|_{\omega}
\end{array}\right.
$$

where $\left.w\right|_{\omega}$ represented the restriction of the state $w$ to $\omega$ [28]. There adjoints are respectively denoted by $X_{\omega}^{*}$ are defined by

$$
x_{\omega}^{*}:\left\{\begin{aligned}
\left(H^{1}(\omega)\right)^{n} & \rightarrow\left(H^{1}(\mho)\right)^{n} \\
w \rightarrow X_{\omega}^{*} w & = \begin{cases}w I_{\omega} & \text { in } \omega \\
0 & \text { in } \mho \backslash \omega\end{cases}
\end{aligned}\right.
$$

- Finally, we introduced the operator $H_{\mathfrak{F}}=X_{\mathfrak{F}} \gamma \nabla \mathcal{K}^{*}$ from $\mathbb{Y}$ into $\left(H^{1 / 2}(\mathfrak{F})\right)^{n}$.

\subsection{Definitions and Descriptions}

This section part presents necessary results about the RBG-Observability notion devoted to a particular devoted sensors. On behalf of this objective, one can deliberate the ADDP-Systems characterizes (1) in the autonomous case via next form.

$$
\left\{\begin{array}{lc}
\frac{\partial w}{\partial t}(\zeta, t)=\Delta w(\zeta, t) & \Pi_{\mathcal{T}} \\
w(\zeta, 0)=w_{0}(\zeta) & \bar{\mho} \\
\frac{\partial w}{\partial v}(\mu, t)=0 & \bar{\Xi}_{\mathcal{T}}
\end{array}\right.
$$

The Problem Solution of $A D D P$ - Systems (4) is obtainable in the following form

$$
w(\zeta, t)=S_{\Delta}(t) w_{0}(\zeta) \quad \text { for all } t \in[0, T]
$$

Definition2.1: $A D D P$-Systems (4) is increased with the measurement function (2) is so-called to be an $E R G-$ Observable in a region $\omega \subset \mho$, if

$$
\operatorname{Im} H_{\omega}=\left(H^{1}(\omega)\right)^{n}
$$

and $A D D P$-Systems (4) is increased with the measurement function (2) is so-called to be an $W R G-$ Observabile if

$\left.\overline{\operatorname{Im} H_{\omega}}=\left(H^{1}(\omega)\right)^{n}\right)$. 
Definition2.2: $A D D P$ - Systems (4) increased with measurement function (2) is so-called to be an $E R B G-$ Observabile in a boundary region $\mathfrak{F} \subset \partial \mho$, if

$$
\operatorname{Im} H_{\mathfrak{F}}=\left(H^{1 / 2}(\mathfrak{F})\right)^{n}
$$

and $A D D P$ - Systems (4) increased with measurement function (2) is so-called to be an WRBG - Observabile if

$$
\left.\overline{\operatorname{Im} H_{\mathfrak{F}}}=\left(H^{1 / 2}(\mathfrak{F})\right)^{n}\right)
$$

Remark2.3: We conclude that, this equation

$$
\overline{\operatorname{Im} H_{\mathfrak{F}}}=\left(H^{1 / 2}(\mathfrak{F})\right)^{n} \Leftrightarrow \operatorname{ker} H_{\mathfrak{F}}^{*}=\{0\} .
$$

Proposition2.4: $A D D P$ - Systems (4) increased with measurement function (2) are ERBG - Observabile if and only if $\exists v>0$, such as that for all $w^{*} \in\left(H^{1 / 2}(\mathfrak{F})\right)^{n}$, then,

$$
\left\|X_{\mathfrak{F}} w^{*}\right\|_{\left(H^{1 / 2}(\mathscr{F})\right)^{n}} \leq v\left\|\mathcal{K} \nabla^{*} \gamma^{*} \chi_{\mathfrak{F}}^{*} w^{*}\right\|_{\mathbb{Y}}
$$

\section{Proof:}

The proof of Proposition2.4 can be deducted via the next overall conclusions [1]. Supposing $E, F$ as well as $G$ be a reflexive Banach spaces and $f \in \mathcal{L}(E, G), g \in \mathcal{L}(F, G)$, then the following properties are analogous

(I) Imf $\subset$ Img.

(II) $\exists v>0$, such that

$$
\left\|f^{*} w^{*}\right\|_{E^{*}} \leq v\left\|g^{*} w^{*}\right\|_{F^{*}}, \text { for all } w^{*} \in G^{*} .
$$

If we apply this outcome, considered $E=G=\left(H^{1 / 2}(\mathfrak{F})\right)^{n}, F=\mathbb{Y}, f=I d_{\left(H^{1 / 2}(\mathscr{F})\right)^{n}}$ and $g=\mathcal{X}_{\mathfrak{F}} \gamma \nabla \mathcal{K}^{*}$. Therefore, we obtain the inequality

$$
\left\|x_{\mathfrak{F}} w^{*}\right\|_{\left(H^{1 / 2}(\mathscr{F})\right)^{n}} \leq v\left\|\mathcal{K} \nabla^{*} \gamma^{*} \mathcal{X}_{\mathfrak{F}}^{*} w^{*}\right\|_{\mathbb{Y}}
$$

Now, the following proposition can be arrived at:

Proposition2.5: If the ADPD - System is ERB - Observable then it is ERBG-Observable.

Proof: The $A D P D-$ System is an $E R B-$ Observable. Therefore $\exists \gamma_{\mathscr{\mho}}>0$, such that for all $w_{0} \in H^{1 / 2}(\mathfrak{F})$, we have

$$
\left\|w_{0}\right\|_{H^{1 / 2}(\mathscr{F})} \leq \gamma_{\mathfrak{F}}\left\|\mathcal{K} \gamma_{0}^{*} \tilde{x}_{\mathfrak{F}}^{*} w_{0}\right\|_{\mathcal{L}^{2}(0, T, \mathbb{Y})}, \text { for all } \gamma_{\mathfrak{F}}>0
$$

Since $\left(H^{1 / 2}(\mathfrak{F})\right)^{n} \subset H^{1 / 2}(\mathfrak{F})$, then

$$
\left\|\gamma \nabla w_{0}\right\|_{\left(H^{1 / 2}(\partial \mho)\right)^{n}}=\left\|w_{0}\right\|_{\left(H^{1 / 2}(\Re)\right)^{n}} \leq\left\|w_{0}\right\|_{H^{1 / 2}(\Im)}, \text { for all } w_{0} \in H^{1 / 2}(\mathfrak{F})
$$

where,

$$
H^{1 / 2}(\mathfrak{F})=\left\{w_{0}: \int_{\mathfrak{F}}\left|w_{0}\right|^{2}<\infty\right\}
$$

and,

$$
\left(H^{1 / 2}(\mathfrak{F})\right)^{n}=\left\{\nabla w_{0}=g_{i}: \int_{\mathfrak{F}}\left|g_{i}\right|^{2}<\infty, g_{i}=\frac{\partial w_{0}}{\partial \zeta_{i}}, \text { for all } i=1,2, \ldots\right\}
$$

So to demonstrate $\left\|w_{0}\right\|_{\left(H^{1 / 2}(\mathscr{F})\right)^{n}} \leq v\left\|\mathcal{K} \nabla^{*} \gamma^{*} \chi_{\mathscr{F}}^{*} w_{0}\right\|_{\mathcal{L}^{2}(0, T, \mathbb{Y})^{\prime}}$, we have, from (7) and since the ADPD - System is $E R B-$ Observable, then there exists $\gamma_{\mathscr{F}}>0$ and $v>0$, such that $\gamma_{\mathscr{F}}=\frac{1}{v}$, by setting

$$
v=\frac{\left\|\mathcal{K} \gamma_{0}^{*} \widetilde{x}_{\mathfrak{F}}^{*} w_{0}\right\|_{\mathbb{Y}}}{\left\|\mathcal{K} \nabla^{*} \gamma^{*} x_{\mathfrak{F}}^{*} w_{0}\right\|_{\mathbb{Y}}}
$$

consequently we can get 


$$
\left\|w_{0}\right\|_{\left(H^{1 / 2}(\mathfrak{F})\right)^{n}} \leq\left\|\mathcal{K} \nabla^{*} \gamma^{*} x_{\mathfrak{F}}^{*} w_{0}\right\|_{\mathbb{Y}^{*}}
$$

Therefore, ADPD - System is ERBG - Observable with $\gamma_{\mathscr{F}}=1$.

\section{Sufficient Conditions For RBGS - Sensors}

For accomplishing the $R B G$-Observability, we must grant the appropriate condition for the characterization of in a specified region $\mathfrak{F}$.

\section{Remark.3.2:}

1- $\operatorname{Sensor}(\mathcal{D}, f)[15]$ may be pointwise if $\mathcal{D}=\{\mathfrak{b}\}$, with $b \in \bar{\mho}$ and $f=\delta(.-\mathfrak{b})$, whereas $\delta$ is the mass of Dirac focused in $b$. Then the measurement output function (2) formulated by [1-3].

$$
y(t)=\int_{\mho} w(\zeta, t) \delta_{\mathfrak{b}}(\zeta-\mathfrak{b}) d \zeta=w(\mathfrak{b}, t)
$$

2- So, in the zone circumstance, $\mathcal{D} \subset \mho$ as well as $f \in \mathcal{L}^{2}(\mathcal{D})$. Hence the measurement function

$$
y(t)=\int_{\mathcal{D}} w(\zeta, t) f(\zeta) d \zeta
$$

Definition3.2: The couple $(\mathcal{D}, f)$ is $R B G S$-Sensor, if the linked $A D P D-$ System is WRBG-Observable.

Definition3.3: Sensors $\left(\mathcal{D}_{i}, f_{i}\right)_{1 \leq i \leq q}$ are $R B G S-$ Sensor, if one of them symbolized by $\left(\mathcal{D}_{1}, f_{1}\right)$ is $R B G S-$ Sensor.

Proposition3.4: The couple $(\mathcal{D}, f)$ is $R B G S$-Sensor if and only $N_{\mathfrak{F}}=H H^{*} \quad$ represent a positive definite operator.

Proof: As $(\mathcal{D}, f)$ is $R B G S$ - Sensor means that the linked $A D P D-$ System is WRBG-Observable. Thus, if $w^{*} \in\left(H^{1 / 2}(\mathfrak{F})\right)^{n}$, achieves the subsequent

$$
\left\langle N_{\mathfrak{\mho}} w^{*}, w^{*}\right\rangle_{\left(H^{1 / 2}(\mathfrak{F})\right)^{n}}=0 \text {, then }\left\|H^{*} w^{*}\right\|_{\mathbb{Y}}=0
$$

Henceforth $w^{*} \in \operatorname{ker} H^{*}$, thus $w^{*}=0$, i.e., $N_{\mathfrak{F}}$ is positive definite.

Conversely, let $w^{*} \in\left(H^{1 / 2}(\mathfrak{F})\right)^{n}$, such that

$$
H^{*} w^{*}=0 \text {, then }\left\langle H^{*} w^{*}, H^{*} w^{*}\right\rangle_{\mathbb{Y}}=0
$$

and thus,

$$
\left\langle N_{\mathfrak{F}} w^{*}, w^{*}\right\rangle_{\left(H^{1 / 2}(\mathscr{F})\right)^{n}}=0 .
$$

Thus $w^{*}=0$, therefore, the linked $A D P D-$ System is $W R B G-$ Observable.

and then, $(\mathcal{D}, f)$ is $R B G S-$ Sensor..

Proposition.3.5: The couple $(\mathcal{D}, f)$ is $R B G S$-Sensor, if the linked $A D P D-S y s t e m$ is ERBG - Observable.

Proof : As the ADPD - System is ERBG - Observable. Thus,

$$
\operatorname{Im} H_{\mathfrak{F}}=\left(H^{1 / 2}(\mathfrak{F})\right)^{n}
$$

As well known $\left(H^{1 / 2}(\partial \mho)\right)^{n}$ is $H S$. So that leads to the form

$$
\operatorname{ker} \mathcal{X}_{\mathfrak{F}}+\operatorname{Im} \mathcal{X}_{\mathfrak{F}}^{*} \mathcal{X}_{\mathfrak{F}} \gamma \nabla \mathcal{K}^{*}=\left(H^{1 / 2}(\partial \mho)\right)^{n}
$$

we obtain that,

$$
\operatorname{ker} \mathcal{K}(t) \nabla^{*} \gamma^{*} \chi_{\mathfrak{F}}^{*}=\{0\}
$$

and this is equivalent to

$$
\overline{\operatorname{Im} \mathcal{X}_{\mathfrak{F}} \gamma \nabla \mathcal{K}^{*}}=\left(H^{1 / 2}(\mathfrak{F})\right)^{n} .
$$

Later, the connected ADPD - System is WRBG - Observable. Consequently, $(\mathcal{D}, f)$ stays RBGS - Sensor. $\square$

Remark.3.6: As of the preceding outcomes, we can realized the next: 
(I) An ADPD - System is EBG - Observable, then the ADPD - System is WRBG-Observable.

$(I I)$ If a couple $(\mathcal{D}, f)$ is $R B G S-$ Sensor in $\mathfrak{F}_{1}$ for an $A D P D-S y s t e m$, then it is $R B G S-$ Sensor in $\mathfrak{F}_{2}$ subregion of $\mathfrak{F}_{1}$.

\subsection{The Main Results}

This part concerns with developing the consequences to the concept of $R B G S$-Sensors in the corresponding $A D P D$ - System, and presents the enough conditions for such sensor. So that, it is assumed that there is $\left(\varphi_{n j}\right)_{n \in I, j=1, \ldots, m_{n}}$ of $\Delta$ in $H^{1}(\bar{\mho})$ denoted $a$ set of eigenfunctions [10], associated with eigenvalue $\lambda_{n}$ of multiplicities $m_{n}$ and $m_{n}=\sup _{n \in I} m_{n}$ is finite. For $\bar{w}=\left(w_{1}, \ldots, w_{n-1}\right)$ and $\bar{n}=\left(n_{1}, \ldots, n_{n-1}\right)$. Suppose that the function $\psi_{\bar{n} j}(\bar{w})=X_{\mathfrak{F}} \gamma \nabla \varphi_{n j}(w), n \in I$, is a complete set in $\left(H^{1 / 2}(\mathfrak{F})\right)^{n}$. If the reflected DDP - System (1) where $J$ satisfy instability property. Thus, the succeeding outcome can be obtained.

Theorem3.7: Suppose that $\sup m_{n}=m<\infty$, then the couples $\left(\mathcal{D}_{i}, f_{i}\right)_{1 \leq i \leq q}$ are RBGS-Sensors iff

1. $q \geq m$,

2. $\operatorname{rank} G_{n}=m_{n}$, for all $n \geq 1$, where $G_{n}=\left(G_{n}\right)_{i j}$ with $1 \leq i \leq q, 1 \leq j \leq m_{n}$, and

$$
\left(G_{n}\right)_{i j}=\left\{\begin{array}{lr}
\sum_{\mathcal{K}=1}^{n} \frac{\partial \psi_{\bar{n} j}}{\partial w_{\mathcal{K}}}\left(\mathfrak{b}_{i}\right) & \text { point wise sensor } \\
\sum_{\mathcal{K}=1}^{n}\left\langle\frac{\partial \psi_{\bar{n} j}}{\partial w_{\mathcal{K}}}, f_{i}\right\rangle_{\mathcal{L}^{2}\left(\mathcal{D}_{i}\right)} & \text { zone sensor }
\end{array}\right.
$$

Proof: First, we evoke that the ADPD - System is WRBG - Observable, this means:

$$
\left[\mathcal{K} \nabla^{*} \gamma^{*} x_{\mathscr{F}}^{*} w^{*}=0 \Rightarrow w^{*}=0\right] .
$$

which allows to state that the couples $\left(\mathcal{D}_{i}, f_{i}\right)_{1 \leq i \leq q}$ are $R B G S$-Sensors iff

$$
\left\{w^{*} \in\left(H^{1 / 2}(\mathfrak{F})\right)^{n} \mid\left\langle H \mathcal{Y}, w^{*}\right\rangle_{\left(H^{1 / 2}(\mathscr{F})\right)^{n}}=0 \text {, for all } \mathcal{Y} \in \mathbb{Y}\right\}=\{0\}[12] \text {. }
$$

By supposing that the couples $\left(\mathcal{D}_{i}, f_{i}\right)_{1 \leq i \leq q}$ are RBGS - Sensors, but for a certain $n \in N$, then, $\operatorname{rank} G_{n} \neq m_{n}$ i.e.:

$$
\forall w_{n}=\left(w_{n_{1}}, w_{n_{2}}, \ldots, w_{n_{m}}\right)^{T} \neq 0,
$$

such that

$$
G_{n} w_{n}=0, w_{0}=\sum_{j=1}^{m_{n}} w_{n j} \psi_{n j} \in H^{1 / 2}(\mathfrak{F}) \neq 0
$$

So, we can rebuild a non-zero $w_{0} \in H^{1 / 2}(\mathfrak{F})$ in considering

$$
\left\langle w_{0}, \psi_{p j}\right\rangle_{H^{1 / 2}(\mathscr{\xi})}=0 \text {. }
$$

If $p \neq n$, and $\left\langle w_{0}, \psi_{n j}\right\rangle_{H^{1 / 2}(\mathscr{\xi})}=w_{n j}, 1 \leq j \leq m_{n}$, then

$$
\begin{aligned}
& \left\langle H \mathcal{Y}, w_{0}\right\rangle_{\left(H^{1 / 2}(\mathscr{F})\right)^{n}}=\sum_{\mathcal{K}=1}^{n}\left\langle\widetilde{x}_{\mathfrak{F}} \gamma_{0} \frac{\partial}{\partial \zeta_{\mathcal{K}}}\left(\mathcal{K}^{*} \mathcal{Y}\right),{\widetilde{x_{F}}}_{\mathfrak{F}} w_{0}\right\rangle_{H^{1 / 2}(\mathfrak{F})} \\
& =\sum_{\mathcal{K}=1}^{n}\left\langle\frac{\partial}{\partial \zeta_{\mathcal{K}}}\left(\widetilde{w}(T), \gamma_{0}{\widetilde{x_{\overparen{F}}^{*}}}_{w_{0}}\right\rangle_{H^{1 / 2}(\partial \mho)}\right.
\end{aligned}
$$

where $\widetilde{w}$ maybe signifies as a solution of the ensuing form

$$
\left\{\begin{array}{lr}
\frac{\partial \widetilde{w}}{\partial t}(\zeta, t)=\Delta^{*} \widetilde{w}(\zeta, t)+\sum_{i=1}^{q} f_{i} \mathcal{Y}_{i}(T-t) & \Pi_{\mathcal{T}} \\
\widetilde{w}(\zeta, 0)=0 & \bar{\mho} \\
\frac{\partial \widetilde{w}}{\partial v}(\mu, t)=0 & \Xi_{\mathcal{T}}
\end{array}\right.
$$

Now, consider the system

$$
\begin{cases}\frac{\partial \psi}{\partial t}(\zeta, t)=-\Delta \varphi(\zeta, t) & \Pi_{\mathcal{T}} \\ \psi(\zeta, 0)=\gamma_{0}^{*} \widetilde{X}_{\Gamma}^{*} w_{0} & \bar{\mho} \\ \frac{\partial \psi}{\partial v}(\mu, t)=0 & \Xi_{\mathcal{T}}\end{cases}
$$


multiply (10) by $\frac{\partial \psi}{\partial \zeta_{\mathcal{K}}}$ and integrate on $\Pi_{\mathcal{T}}$, we get that

$$
\begin{aligned}
\int_{\Pi_{\mathcal{T}}} \frac{\partial \psi}{\partial \zeta_{\mathcal{K}}}(\zeta, t) \frac{\partial \widetilde{w}}{\partial t}(\zeta, t) d \zeta d t= & \int_{\Pi_{\mathcal{T}}} \Delta^{*} \widetilde{w}(\zeta, t) \frac{\partial \psi}{\partial \zeta_{t}}(\zeta, t) d \zeta d t \\
& +\int_{\Pi_{\mathcal{T}}}\left(\sum_{i=1}^{q} \delta_{b_{i}} y_{i}(T-t)\right) \frac{\partial \psi}{\partial \zeta_{\mathcal{K}}}(\zeta, t) d \zeta d t
\end{aligned}
$$

But, we have

$$
\int_{\Pi_{J}} \frac{\partial \varphi}{\partial \zeta_{\mathcal{K}}}(\zeta, t) \frac{\partial \widetilde{w}}{\partial t}(\zeta, t) d \zeta d t=\int_{\partial \mho}\left[\frac{\partial \varphi}{\partial \zeta_{\mathcal{K}}}(\zeta, t) \widetilde{w}(\zeta, t) d \zeta\right]_{0}^{T}+
$$

$\int_{\Pi_{\mathcal{T}}} \Delta \frac{\partial \psi}{\partial \zeta_{\mathcal{K}}}(\zeta, t) \widetilde{w}(\zeta, t) d \zeta d t \int_{\partial \mho} \frac{\partial \psi}{\partial \zeta_{\mathcal{K}}}(\zeta, t) \widetilde{w}(\zeta, t) d \zeta+\int_{\Pi_{\mathcal{T}}} \Pi \frac{\partial \psi}{\partial \zeta_{\mathcal{K}}}(\zeta, t) \widetilde{w}(\zeta, t) d \zeta d t$.

then,

$$
\begin{aligned}
& \int_{\partial \mho} \frac{\partial \psi}{\partial \zeta_{\mathcal{K}}}(\zeta, t) \widetilde{w}(\zeta, t) d \zeta=-\int_{\Pi_{\mathcal{T}}} \Pi_{\mathcal{T}} \frac{\partial \psi}{\partial \zeta_{\mathcal{K}}}(\zeta, t) \widetilde{w}(\zeta, t) d \zeta \\
& +\int_{\Pi_{\mathcal{T}}} \Delta^{*} \widetilde{w}(\zeta, t) \frac{\partial \psi}{\partial \zeta_{\mathcal{K}}}(\zeta, t) d \zeta d t+\int_{\Delta}\left(\sum_{i=1}^{q} \delta_{b_{i}} y_{i}(T-t)\right) \frac{\partial \psi}{\partial \zeta_{\mathcal{K}}}(\zeta, t) d \zeta d t
\end{aligned}
$$

Integrating by parts, we obtain

$$
\begin{aligned}
\int_{\partial \mho} \frac{\partial \psi}{\partial \zeta_{\mathcal{K}}}(\zeta, t) \widetilde{w}(\zeta, t) d \zeta= & -\int_{\Pi_{\mathcal{T}}} \frac{\partial \widetilde{w}(\mu, t)}{\partial v_{\Delta^{*}}} \frac{\partial \psi}{\partial \zeta_{\mathcal{K}}}(\mu, t) d \\
& +\int_{\Pi_{\mathcal{T}}} \frac{\partial}{\partial v_{\Delta^{*}}}\left(\frac{\partial \psi}{\partial \zeta_{\mathcal{K}}}(\mu, t) d \mu d t\right) \widetilde{w}(\mu, t) \\
& +\int_{\Pi_{\mathcal{T}}}\left(\sum_{i=1}^{q} \delta_{b_{i}} y_{i}(\mathcal{T}-t)\right) \frac{\partial \psi}{\partial \zeta_{\mathcal{K}}}(\zeta, t) d \zeta d t .
\end{aligned}
$$

the boundary conditions give

$$
\int_{\partial \mho} \frac{\partial \psi}{\partial \zeta_{\mathcal{K}}}(\zeta, t) \widetilde{w}(\zeta, t) d \zeta=\int_{\Pi}\left(\sum_{i=1}^{q} \delta_{b_{i}} y_{i}(T-t)\right) \frac{\partial \psi}{\partial \zeta_{\mathcal{K}}}(\zeta, t) d \zeta d t .
$$

Thus,

$$
\int_{\partial \mho} \psi(\zeta, t) \frac{\partial \widetilde{w}}{\partial \zeta_{\mathcal{K}}}(\zeta, T) d \zeta=-\sum_{i=1}^{q} \int_{0}^{T} \frac{\partial \psi}{\partial \zeta_{\mathcal{K}}}\left(\mathfrak{b}_{i}, t\right) \mathcal{Y}_{i}(\mathcal{T}-t) d t
$$

and, we have

$$
\begin{aligned}
\left\langle\chi_{\mathfrak{F}} \gamma \nabla \mathcal{K}^{*}, w_{0}\right\rangle_{\left(H^{\left.\frac{1}{2}(\mathscr{Y})^{n}\right)}\right.}= & \sum_{\mathcal{K}=1}^{n} \int_{\mho} \frac{\partial \widetilde{w}}{\partial \zeta_{\mathcal{K}}}(\zeta, t) \psi(\zeta, t) d \zeta= \\
& -\sum_{\mathcal{K}=1}^{q} \int_{0}^{T} \sum_{\mathcal{K}=1}^{n} \frac{\partial \psi}{\partial \zeta_{\mathcal{K}}}\left(\mathrm{b}_{i}, t\right) y_{i}(\mathcal{T}-t) d t
\end{aligned}
$$

but,

$$
\psi(\zeta, t)=\sum_{p=1}^{\infty} e^{-\lambda_{p}(\mathcal{T}-t)} \sum_{j=1}^{m_{p}}\left\langle w_{0}, \psi_{p j}\right\rangle_{\mathcal{L}^{2}(\omega)} \psi_{p j}
$$

Then,

$$
\begin{aligned}
\sum_{\mathcal{K}=1}^{n} \frac{\partial \psi}{\partial \zeta_{\mathcal{K}}}\left(\mathfrak{b}_{i}, t\right)= & \sum_{p=1}^{\infty} e^{-\lambda_{p}(\mathcal{T}-t)} \sum_{j=1}^{m_{p}}\left\langle w_{0}, \varphi_{p j}\right\rangle_{\mathcal{L}^{2}(\omega)} \sum_{\mathcal{K}=1}^{n} \frac{\partial \psi}{\partial \zeta_{\mathcal{K}}}\left(\mathfrak{b}_{i}\right) \\
& =\sum_{p=1}^{\infty} e^{\lambda_{p}(\mathcal{T}-t)}\left(G_{p} w_{p}\right)_{i}
\end{aligned}
$$

therefore,

$$
\left\langle\mathcal{X}_{\mathfrak{F}} \gamma \nabla \mathcal{K}^{*} \mathcal{Y}, w_{0}\right\rangle_{\left(H^{1 / 2}(\mathscr{F})\right)^{n}}=-\sum_{\mathcal{K}=1}^{q} \int_{0}^{T} \sum_{p=1}^{\infty} e^{\lambda_{p}(\mathcal{T}-t)}\left(G_{p} w_{p}\right) w_{i}(\mathcal{T}-t) d t
$$

thus,

$$
\left\langle\mathcal{X}_{\mathfrak{F}} \gamma \nabla \mathcal{K}^{*} \mathcal{Y}, w_{0}\right\rangle_{\left(H^{1 / 2}(\mathfrak{F})\right)^{n}}=-\sum_{i=1}^{q} \int_{0}^{T} e^{\lambda_{n}(\mathcal{T}-t)}\left(G_{n} w_{n}\right)_{i} \mathcal{Y}_{i}(\mathcal{T}-t) d t=0,
$$

$\forall \mathcal{Y} \in \mathcal{L}^{2}\left(0, \mathcal{T}, R^{q}\right)$. 
Subsequently, $w_{0} \in$ ker $\mathrm{H}_{\mathfrak{F}}^{*}$ this is conflicted to the hypotheses. Thus, $\left(\mathcal{D}_{i}, f_{i}\right)_{1 \leq i \leq q}$ are RBGS - Sensors for the ADPD - System (4)

\subsection{Internal and $R B G$-Reconstruction via Internal Region}

$R B G S$ - Sensors problem for ADPD - System may be seen as internal RGS - Sensors, if we deliberate $\bar{\omega}_{r} \subset$ $\bar{\mho}[27]$.

- Let $\Re$ is an linear operator of extension continuous type which is represented via

$\mathfrak{R}:\left(H^{1 / 2}(\partial \mho)\right)^{n} \rightarrow\left(H^{1}(\mho)\right)^{n}$, such that

$\gamma \nabla \Re h(\zeta, t)=h(\zeta, t)$, for all $h(\zeta, t) \in\left(H^{1 / 2}(\partial \mho)\right)^{n}$

- For $r>0$ any real number such that satisfactorily small we can define

$E=\bigcup_{w \in \mathscr{F}} B(w, r), \bar{\omega}_{r}=E \cap \mho$ and $\mathfrak{F}=\bar{\omega}_{r} \cap \partial \mho$,

where $B(w, r)$ is a ball radius $r$ focused in $w(\zeta, r)$, so $\mathfrak{F}$ is a subregion of $\bar{\omega}_{r}$ (Figure. 2).

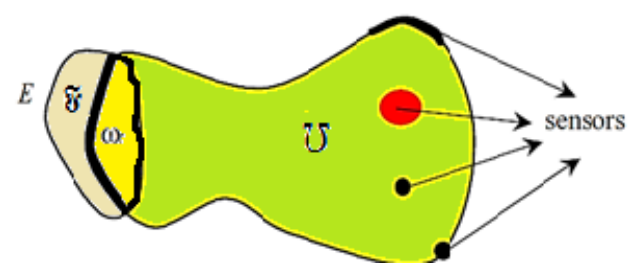

Fig.2: Internal region $\omega_{r}$ and boundary $\mathfrak{F}$.

In the next consequences, we demonstrate that the link between the RBGS - Sensors problem and $\bar{\omega}_{r} G S-$ Sensors.

\section{Proposition 3.8:}

(I) If the couples $\left(\mathcal{D}_{i}, f_{i}\right)_{1 \leq i \leq q}$ are $\bar{\omega}_{r} G S-$ Sensors in ADPD - System, then, there are RBGS - Sensors.

(II) If the $A D P D-$ System is $E \bar{\omega}_{r} G-$ Observable then, the couples $\left(\mathcal{D}_{i}, f_{i}\right)_{1 \leq i \leq q}$ then, there are RBGSSensors.

Remark.3.9: As of the preceding outcomes, then, we have:

(I) If the $A D P D-$ System is $E \bar{\omega}_{r} G-$ Observable, then it is $E R B G-$ Observable, i.e., $\exists \mathcal{X}_{\bar{\omega}_{r}} \nabla \mathcal{K}^{*}: \mathbb{Y} \rightarrow\left(H^{1}\left(\omega_{r}\right)\right)^{n}$ an operator given by

$$
H_{\bar{\omega}_{r}} Y(., t)=X_{\bar{\omega}_{r}} \nabla \mathcal{K}^{*} Y(., t)=X_{\bar{\omega}_{r}} \Re \bar{w}(\zeta, t) .
$$

Hence,

$$
x_{\mathscr{F}}\left(\gamma x_{\bar{\omega}_{r}} \nabla \mathcal{K}^{*} \mathcal{Y}(., t)\right)=w(\zeta, t)
$$

where $w(\zeta, t) \in\left(H^{1 / 2}(\mathfrak{F})\right)^{n}$ and $\bar{w}(\zeta, t)$ be an extension to $\left(H^{1 / 2}(\partial \mho)\right)^{n}$.

(II) If the $A D P D-$ System is $W \bar{\omega}_{r} G-$ Observable, then it is $W R B G-$ Observable.

(III) An development of the outcomes can be employed for diverse issues of $R G$-Observability [5, 29], and to the $R B G$ - Observability ) of asymptotic reduced case in ADPD - Systems [7].

\section{Applications Of Some Sensor Locations}

This part is devoted to the application of these outcomes for ADPD-System described in $\mho=$ ] $0, a_{1}[\times] 0, a_{2}[$, via the form 


$$
\left\{\begin{array}{lr}
\frac{\partial w}{\partial t}\left(\zeta_{1}, \zeta_{2}, t\right)=\frac{\partial^{2} w}{\partial \zeta_{1}^{2}}\left(\zeta_{1}, \zeta_{2}, t\right)+\frac{\partial^{2} w}{\partial \zeta_{2}^{2}}\left(\zeta_{1}, \zeta_{2}, t\right)+w\left(\zeta_{1}, \zeta_{2}, t\right) & \Pi_{\mathcal{T}} \\
w\left(\zeta_{1}, \zeta_{2}, 0\right)=w_{0}\left(\zeta_{1}, \zeta_{2}\right) & \bar{\mho} \\
\frac{\partial w}{\partial v}\left(\mu_{1}, \mu_{2}, t\right)=0 & \bar{\Xi}_{\mathcal{T}}
\end{array}\right.
$$

where $\mathfrak{F}=] 0, a_{2}\left[, \times\left\{a_{2}\right\}\right.$ or $\left.\mathfrak{F}=\left\{a_{1}\right\} \times\right] 0, a_{2}[$, the eigenfunctions of the system (13) is given by

$$
\varphi_{n m}\left(\zeta_{1}, \zeta_{2}\right)=\frac{2}{\sqrt{a_{1} a_{2}}} \cos n \pi \frac{\zeta_{1}}{a_{1}} \cos m \pi \frac{\zeta_{2}}{a_{2}}
$$

associated with eigenvalues

$$
\lambda_{n m}=-\frac{n^{2} \pi^{2}}{a_{1}^{2}}-\frac{m^{2} \pi^{2}}{a_{2}^{2}}, n, m \geq 1
$$

If we assume that $a_{1}^{2} / a_{2}^{2} \notin Q$, and hence $\lambda_{n m}$ is the multiplicity of $r_{n m}=1$. Consequently the couple $(\mathcal{D}, f)$ may be enough to realize $R B G$ - Observability of the observed ADPD - System as in [3-6] . Now, in the following outcomes give information on the location of (pointwise and zone) RBGS - Sensors.

\section{1 Sensor of Zone Type}

This sub-section will be devoted to study the subsequent cases.

\subsection{Case Figure3}

Take into consideration the $A D P D$ - System (13) with the measurement equation (2) which is formulated via

$$
y(t)=\int_{\mathcal{D}} w\left(\zeta_{1}, \zeta_{2}, t\right) f\left(\zeta_{1}, \zeta_{2}\right) d \zeta_{1} d \zeta_{2}
$$

with the couple $(\mathcal{D}, f)$ sensor of type zone is placed in the domain $\mho$, done the supports $\mathcal{D}=] \zeta_{1}-\ell_{1}, \zeta_{1}+\ell_{1}[\times] \zeta_{2}-\ell_{2}, \zeta_{2}+\ell_{2}[\in \mho$ as in (Figure3).

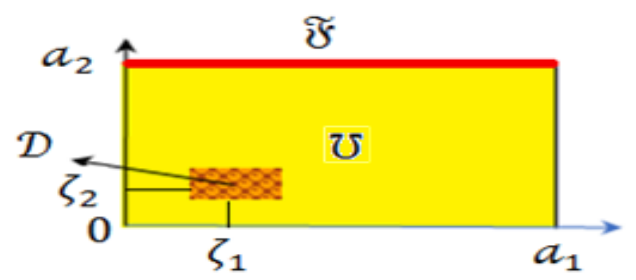

Fig. 3: Internal zone sensor $\mathcal{D}$.

Then, we have the subsequent consequence.

Proposition 4.1: If $f$ satisfies symmetry property with around to $\zeta=\left(\zeta_{1}, \zeta_{2}\right)$, so the couple $(\mathcal{D}, f)$ is $R B G S-$ Sensor in $\mathfrak{F}=] 0, a_{2}\left[\times\left\{a_{2}\right\}\right.$ for the ADPD - System $(13-16)$, if

$$
\frac{n_{0} \zeta_{1}}{a_{1}} \text { and } \frac{m_{0} \zeta_{2}}{a_{2}} \in \mathcal{Q} \text {, for all } n_{0}, m_{0}=\{1, \ldots, J\} \text {. }
$$

\section{1.2 Boundary Zone Case}

We discuss this case as the follows:

\section{Case of Figure 3}

In this case, where $\mathfrak{F}_{0}=\left[\mu_{10}-\ell_{1}, \mu_{1_{0}}+\ell_{1}\right] \times\left\{a_{2}\right\}$ is the support of the boundary sensor and $f \in \mathcal{L}^{2}\left(\mathfrak{F}_{0}\right)$ as in (Figure4), 


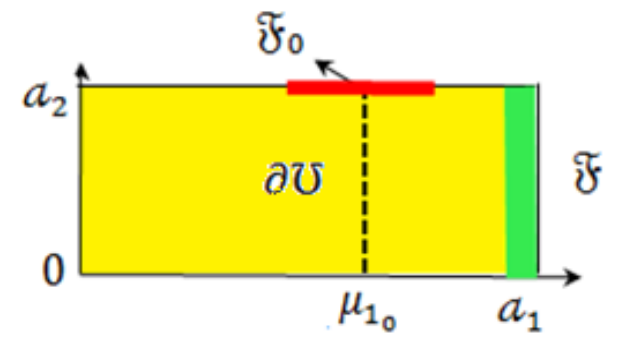

Fig. 4: One side boundary zone sensor $\mathfrak{F}_{0}$.

The measurements are shown by the output function

$$
y(t)=\int_{\widetilde{F}_{0}} \frac{\partial w}{\partial v}\left(\mu_{1}, \mu_{2}, t\right) f\left(\mu_{1}, \mu_{2}\right) d \mu_{1} \mu_{2}
$$

Then, we arrive to the result:

\section{Proposition 4.2:}

Assume that the sensors ( $\left.\mathfrak{F}_{0}, f\right)$ are located on $\mathfrak{F}_{0} \subset \partial \mho$ and $f$ is symmetric with respect to $\mu_{1}=\mu_{1_{0}}$, then the couple $\left(\mathfrak{F}_{0}, f\right)$ is $R B G S-$ Sensor in $\left.\mathfrak{F}=\left\{a_{1}\right\} \times\right] 0, a_{2}[\subset \partial \mho$ for the ADPD - System $(13-17)$, if

$$
\frac{n \mu_{1} 0}{a_{1}} \notin Q, \text { for all } n=\{1, \ldots, J\} \text {. }
$$

\section{Figure5 case}

In this case, where $\overline{\mathfrak{F}} \subset \partial \mho$ is the support of the boundary sensor and $f \in \mathcal{L}^{2}(\overline{\mathfrak{F}})$ as in (Figure5).

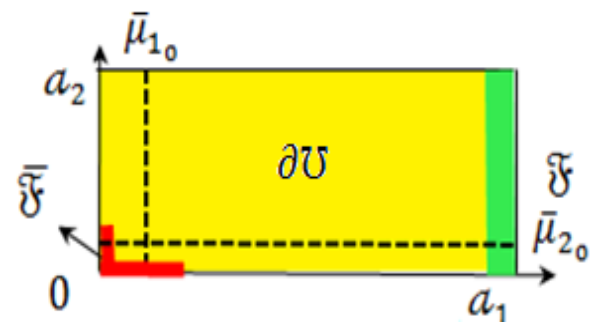

Fig. 5: Both sides boundary zone sensor $\overline{\mathfrak{F}}$.

Now, $\left.\mathfrak{F}=\left\{a_{1}\right\} \times\right] 0, a_{2}[\subset \partial \mho$ is the observed region and the measurements are shown by the output

$$
\mathcal{Y}(t)=\int_{\mathscr{\mathscr { F }}} \frac{\partial w}{\partial v}\left(\mu_{1}, \mu_{2}, t\right) f\left(\mu_{1}, \mu_{2}\right) d \mu_{1} \mu_{2}
$$

Then, we reach to the subsequent consequence.

\section{Proposition4.3:}

Suppose that $(\overline{\mathscr{F}}, f)$ to be the situated sensors on $\overline{\mathscr{F}}_{\mathfrak{F}}=\overline{\mathscr{F}}_{1} \cup \overline{\mathfrak{F}}_{2}=\left[0, \bar{\mu}_{10}+\ell_{1}\right] \times\{0\} \cup\{0\} \times\left[0, \bar{\mu}_{20}+\ell_{2}\right] \subset$ $\partial \mho$ and $\left.f\right|_{\bar{\mho}_{1}}$ is symmetric around to $\bar{\mu}_{1}=\bar{\mu}_{1_{0}}$ and $\left.f\right|_{\bar{\mho}_{2}}$ is symmetric around to $\bar{\mu}_{2}=\bar{\mu}_{20^{\prime \prime}}$ then the couple $(\overline{\mathscr{F}}, f)$ is RBGS - Sensor on $\overline{\mathfrak{F}}$ for the ADPD - System $(13-18)$, if

$$
\frac{n \bar{\mu}_{1}}{a_{1}} \text { and } \frac{m \bar{\mu}_{20}}{a_{2}} \notin \mathcal{Q}, \text { for all } n, m=\{1, \ldots, J\},
$$

This indicates that the (RBG - observability) relies on the sensors support shape and measurements equation.

\section{2 Sensor of Pointwise type}

This sub-section is devoted for discussing and describing the RBGS-Sensor on $\mathfrak{F}$ for the ADPD - System indifferent situations.

\section{2. 1.Internal Pointwise Sensor}

In this situation, we have two cases: 


\section{(I) Pointwise case:}

The output equation described by

$$
y(t)=\int_{\mho} w\left(\zeta_{1}, \zeta_{2}, t\right) \delta\left(\zeta_{1}-\mathfrak{b}_{1}, \zeta_{2}-\mathfrak{b}_{2}\right) d \zeta_{1} d \zeta_{2}
$$

where $\mathfrak{b}=\left(\mathfrak{b}_{1}, \mathfrak{b}_{2}\right)$ is the sensor pointwise position in $\mho=\left[0, a_{1}\right] \times\left[0, a_{2}\right]$ as defined in (Figure6).

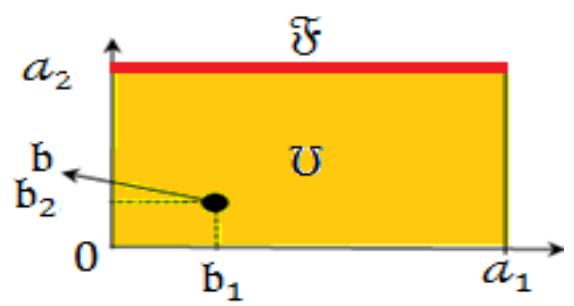

Fig. 6: Internal pointwise sensor b.

Proposition 4.4: If $n \mathfrak{b}_{1} / a_{1}$ and $m b_{2} / a_{2} \notin Q$, for all $n, m=\{1, \ldots, J\}$, then the couple $\left(\mathfrak{b}, \delta_{\mathfrak{b}}\right)$ is $R B G S-$ Sensor on $\mathfrak{F}=] 0, a_{2}\left[\times\left\{a_{2}\right\}\right.$ for the ADPD - System $(13-19)$.

\section{(II) Filament case:}

Deliberate the case where the measurement information is given via the curve $\beta=\operatorname{Im}(\rho)$ such that $\rho \in \mathbb{C}^{1}(0,1)$ (Figure7).

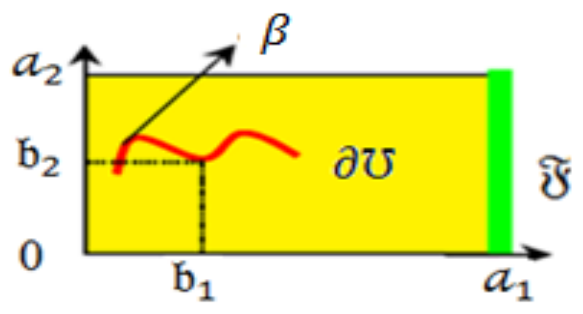

Fig. 7: Internal filament sensor $\beta$.

Proposition 4.5: Assume that the $\beta$ satisfy summitry property around line filament $\mathfrak{b}=\left(\mathfrak{b}_{1}, \mathfrak{b}_{2}\right)$, if $n \mathfrak{b}_{1} / a_{1}$ and $m \mathfrak{b}_{2} / a_{2} \notin Q$, for all $n, m=\{1, \ldots, J\}$, then the couple $\left(\beta, \delta_{\rho}\right)$ is $R B G S-$ Sensor on $\mathfrak{F}=$ ] $0, a_{2}\left[\times\left\{a_{2}\right\}\right.$ for the ADPD - System $(13-19)$.

\section{2. 2.Boundary Pointwise Sensor}

Assume that the sensor $\left(\mathfrak{b}, \delta_{\mathfrak{b}}\right)$ is placed on $\mathfrak{b}$, where $\mathfrak{b}=\left(\mathfrak{b}_{1}, \mathfrak{b}_{2}\right) \in \partial \mho$ such that $\mathfrak{b}=\left(0, \mathfrak{b}_{2}\right)$ by way of (Figure8 $)$.

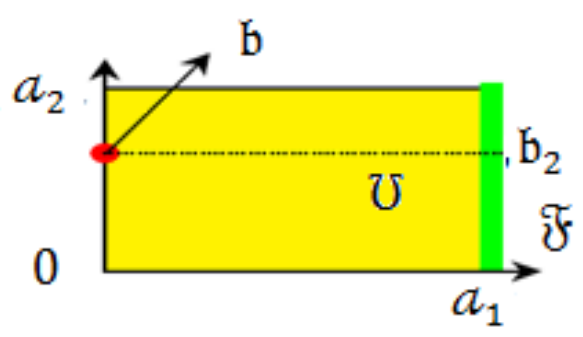

Fig. 8: Boundary pointwise sensor b.

The output function is got by

$$
y(t)=\int_{\partial \mho} w\left(\mu_{1}, \mu_{2}, t\right) \delta\left(0, \mu_{2}-\mathfrak{b}_{2}\right) d \mu_{1} d \mu_{2}
$$

Therefore, we acquire the subsequent outcomes. 


\section{Proposition 4.6:}

The couple $\left(\beta, \delta_{\rho}\right)$ is RBGS - Sensor on $\left.\mathfrak{F}=\right] 0, a_{2}\left[\times\left\{a_{2}\right\}\right.$ for the ADPD - System $(13-20)$, if $\frac{m \mathrm{~b}_{2}}{a_{2}} \notin \mathcal{Q}$, for all $m=\{1, \ldots, J\}$.

\section{Conclusions}

This work has been tackled RBGS- sensors concept for the ADPD-System under which situation accomplishes the unknown gradient of the initial state. Additionally the associations of WRBG- observability and ERBGobservability notions have been deliberated and examined in a region $\mathfrak{F}$. So, for DDP-Systems in HS, many remarkable consequences concerning the choice of sensor constructing which are demonstrated in discreet results. Finally, we have specified that there is a linking between the RBGS- sensor with number, sensors characters and related domains. Many complications are not treated, the likelihood to develop these outcomes to the case of $H S$ in quasi forms.

\section{Conflict of interest}

The authors declare that they have no conflict of interest.

\section{Acknowledgments}

Our thanks in advance to the editors and experts for their efforts.

\section{Funding Statement:}

The research is self-sponsored by the authors.

\section{REFERENCES}

1. Al-Saphory R.( 2002). Asymptotic regional boundary observer in distributed parameter systems via sensors structures, Sensors,2,137-152. https://doi.org/10.3390/s20400137

2. Al-Saphory R. (2011). Analyse régionale asymptotique d'une classe de systèmes distribués, Google Book, Amazon Publisher, New York, USA https://books.google.iq/books/about/Analyse r\%C3\%A9gionale asymptotique d une cl.html?id=Trwj OgAACAAJ\&redir esc $=y$

3. Al-Saphory R. (2011). Strategic sensors and regional exponential observability, ISRN Applied Mathematics, Article ID 673052,1-13. https://www.hindawi.com/journals/isrn/2011/673052/

4. Al-Saphory R. Al-Jawari N. and Al-Janabi A. (2016). General asymptotic regional gradient observer, Aust. J. Basic \& Appl. Sci.,10 (9), 8-18. http://www.ajbasweb.com/old/ajbas May 2016.html

5. Al-Saphory R., Al-Jawari, N., Al-Janabi, A.( 2016). Asymptotic regional gradient full-order observer in distributed parabolic systems, International Journal of Contemporary Mathematical Sciences, 11(7), 343 - 358. http://dx.doi.org/10.12988/ijcms.2016.6633

6. Al-Saphory R., Al-Jawari N. and Al-Janabi A. (2021). Asymptotic regional gradient reduced-order observer, Journal of Physics: Conference Series, Preprinted.

7. Al-Saphory R. Al-Jawari N. and Al-Qaisi A. (2010). Regional gradient detectability for infinite dimensional systems, Tikrit Journal of Pure Science,15(2), 1-6. https://www.researchgate.net/publication/261550172

8. [8] Al-Saphory R. and El Jai A. (2001). Sensors characterizations for regional boundary detectability in distributed parameter systems, Sensors and Actuators A,94 (1-2),1-10. https://www.sciencedirect.com/science/article/abs/pii/S0924424701006690

9. Al-Saphory R. and El Jai A.(2001). Sensors and asymptotic $\omega$-observer for distributed diffusion systems, Sensors, 1, 161-182. https://www.mdpi.com/1424-8220/1/5/161/htm

10. Al-Saphory R. and El Jai, A. (2002). Regional asymptotic state reconstruction, international journal of system science,33, 1025-1037. https://www.tandfonline.com/doi/abs/10.1080/00207720210166998

11. [Al-Saphory R., Khalid Z. and Jasim M. (2021). Junction interface conditions for asymptotic gradient full-order observer in Hilbert space, Italian Journal for Pure Science and Applied Mathematics, Preprinted. 
12. Al-Saphory R., Khalid Z. and El-Jai A. (2020). Regional boundary gradient closed loop control system and $\Gamma^{*} A G O F$-observer, Journal of Physics: Conference Series,1664 (012061), 1-20. https://iopscience.iop.org/article/10.1088/1742-6596/1664/1/012061

13. Al-Saphory R., Al-Shaya A. and Rekkab S. (2020).,, Regional boundary asymptotic gradient reduced order observer, Journal of Physics: Conference Series,1664 (012101), 1-19. https://iopscience.iop.org/article/10.1088/1742-6596/1664/1/012101/meta

14. Ben Hadid S., Rekkab S. and Zerrik, E. (2012). Sensors and regional gradient observability of hyperbolic systems, Intelligent Control and Automation,3, 78-89. https://m.scirp.org/papers/17578

15. Brezis H. (1987). Analyse Fonctionnalle, Theorie et Applications, $2^{\mathrm{em}}$ tirage. Masson, Paris, France. https://www.dunod.com/sciences-techniques/analyse-fonctionnelle-theorie-et-applications

16. Curtain R. F. and Pritchard A. J.( 1978). Infinite dimension linear theory systems, Lecture Notes in Control and Information Sciences, Springer-Verlag,8. https://www.springer.com/gp/book/9783540089612

17. Curtain R. F. and Zwart H. (1995), An introduction to infinite dimensional linear system theory, SpringerVerlag, New York. https://www.springer.com/gp/book/9780387944753

18. El Jai A. and Amouroux M. (1988). Sensors and observers in distributed parameter systems, International $\begin{array}{lllll}\text { Journal of } & \text { Control,47 } & \text { (1), } & 333- & 347 .\end{array}$ https://www.tandfonline.com/doi/abs/10.1080/00207178808906013

19. El Jai A., Amouroux, M. and Zerrik, E. (1994). Regional observability of distributed systems, International $\begin{array}{lllll}\text { Journal of } & \text { Systems } & \text { 301-313. }\end{array}$ https:/www.tandfonline.com/doi/abs/10.1080/00207729408928961

20. El Jai A. and Hamzauoi H. (2009). Regional observation and sensors, International Journal of Applied $\begin{array}{lllll}\text { Mathematics and } \quad \text { Computer } & \text { 5-14. }\end{array}$ https://www.sciencedirect.com/science/article/abs/pii/092442479380204T

21. El Jai A. and Pritchard A. (1987). Sensors and actuators in distributed systems, International Journal of Control,46 1139-1153. https://www.tandfonline.com/doi/abs/10.1080/00207178708933956?journalCode=tcon20

22. El Jai A. and Pritchard A. (1988). Sensors and controls in the analysis of distributed parameter systems, Ellis Horwood Series in Mathematics and Applications, Wiley, New York. https://www.ebay.co.uk/itm/Sensors-and-Controls-in-the-Analysis-of-Distributed-SystemsMathematics-and-its/153079971972?epid=91726288\&hash=item23a4470084:i: 153079971972

23. El Jai A., Simon, M.C. and Zerrik, E.( 1993). Regional observability and sensor structures, Sensors and Actuators A.39 (2), 95-102. https://www.sciencedirect.com/science/article/abs/pii/092442479380204T

24. El Jai A., Simon, M.C., Zerrik, E. and Amouroux, M. (1995). Regional observability of a thermal process, IEEE Transaction on Automatic Control.40 (1), 518-521. https:/ieeexplore.ieee.org/document/376073

25. Jasim M. Swady R. and Al-Saphory R. (2013). Dynamical stability of charged isentropic superdense star model, Journal of Mathematical and Computational Science,3 (1), 266-277. http://scik.org/index.php/jmcs/article/view/767

26. Zerrik E., Badraoui L. and El Jai A. (1999). Sensors and regional boundary state reconstruction of parabolic $\begin{array}{llllll}\text { systems, Sensors and Actuators } & \text { 102-117. }\end{array}$ https://www.sciencedirect.com/science/article/abs/pii/S0924424798002933

27. Zerrik E. and Bourray H.( 2003). Gradient Observability for diffusion systems, International Journal of Applied Mathematics and Computer 139-150. http://pldml.icm.edu.pl/pldml/element/bwmeta1.element.bwnjournal-article-amcv13i2p139bwm

28. Zerrik E., Bourray H. and Badraoui L. (2000). How to reconstruct a gradient for parabolic systems, Conference of MTNS 2000, Perpignan, France, June 19-23. https://www.researchgate.net/publication/272505683 